\title{
A Review of Multimodal Medical Image Fusion Techniques
}

\author{
Bing Huang, ${ }^{1}$ Feng Yang $\mathbb{D}^{1,2}$ Mengxiao Yin, ${ }^{1,2}$ Xiaoying Mo, ${ }^{1}$ and Cheng Zhong $\mathbb{D}^{1,2}$ \\ ${ }^{1}$ School of Computer and Electronics and Information, Guangxi University, Nanning, Guangxi 530004, China \\ ${ }^{2}$ Guangxi Key Laboratory of Multimedia Communications Network Technology, China
}

Correspondence should be addressed to Feng Yang; fyang@foxmail.com

Received 9 December 2019; Revised 26 February 2020; Accepted 3 April 2020; Published 23 April 2020

Academic Editor: Maria E. Fantacci

Copyright (c) 2020 Bing Huang et al. This is an open access article distributed under the Creative Commons Attribution License, which permits unrestricted use, distribution, and reproduction in any medium, provided the original work is properly cited.

The medical image fusion is the process of coalescing multiple images from multiple imaging modalities to obtain a fused image with a large amount of information for increasing the clinical applicability of medical images. In this paper, we attempt to give an overview of multimodal medical image fusion methods, putting emphasis on the most recent advances in the domain based on (1) the current fusion methods, including based on deep learning, (2) imaging modalities of medical image fusion, and (3) performance analysis of medical image fusion on mainly data set. Finally, the conclusion of this paper is that the current multimodal medical image fusion research results are more significant and the development trend is on the rise but with many challenges in the research field.

\section{Introduction}

Since the emergence of image fusion in 1985, image information fusion has developed rapidly on military and civilian fields, especially image fusion of infrared and visible light, material analysis, remote sensing image fusion, multifocus image, and medical image fusion. Imaging technology plays an important role in medical diagnosis. The limited information provided by single modal medical images cannot meet the need of clinical diagnosis which requires a large amount of information, making medical image fusion research become a hot field. Medical image fusion can be divided into single-mode fusion and multimodal fusion. Due to the limitation of the information presented by single-modal fusion, there are many researchers engaging in the study of multimodal fusion.

In the field of medical image, imaging techniques such as Computed Tomography (CT), Magnetic Resonance Imaging (MRI), Positron Emission Tomography (PET), and SinglePhoton Emission Computed Tomography (SPECT) have provided clinicians with information of the human body's structural characteristics, soft tissue, and so on. Different imaging methods keep different characteristics, and different sensors obtain different imaging information of the same part. The purpose of the fusion is to obtain better contrast, fusion quality, and perceived experience. The result of the fusion should meet the following conditions: (a) the fused image should retain the information of source images completely; (b) the fused image should not produce any synthetic information, such as artifacts; and (c) bad states should be avoided, such as misregistration and noise [1].

Traditional medical image fusion methods are divided into spatial domain and transform domain. The medical image fusion methods based on spatial domain were the hotspot of the earliest research. The typical methods are principal analysis and HIS. However, spatial domain technology produces spectral distortion and spatial distortion of fused images [2]. For better fusion effects, researchers turn their research focus to the transform domain. It transforms the source image into the frequency domain or other domains to fuse them and then performs reconstruction operations. The fusion process is divided into four levels, namely, signal, feature, symbol, and pixel level [3]. The pixel level is widely used nowadays, and its typical representatives include contour transformation, discrete wavelet transform, and pyramid transform. The transform domain-based method has the advantages of good structure and avoiding distortion, but it also generates noise during the fusion processing. Therefore, denoising is also a challenge for image fusion $[4,5]$. From the papers of the past two years, it can be seen that there is 
almost no method for the proposed fusion algorithm to using spatial domain alone. However, there are many new methods that combine spatial domain methods with transform domain, such as PCA-DWT [6]. With the advent of the deep learning boom, a medical image fusion method based on deep learning emerged in 2017. In recent years, convolutional neural network $(\mathrm{CNN})$, recurrent neural network $(\mathrm{RNN})$, U-Net network, GAN, and other deep learning models have been widely used in medical image registration and segmentation, while only $\mathrm{CNN}$ and U-Net network have been applied into medical image fusion. Convolutional neural network is a kind of neural network for image process, which is composed of convolutional layer, pooling layer, and fully connected layer. Deep learning framework for medical image fusion includes Caffe, Tensorflow, MatConvNet, and the like. At present, U-Net network is found to be trained on Pytorch deep learning framework.

From 2012 to August 2019, medical image fusion technology has been developed significantly, as shown in Figure 1: the number of published papers of medical image fusion has remarkably boomed in the recent years (medical image fusion publications were counted by web of science, from 2012 to August 2019).

The purpose of this review is to summarize the research progresses and future development of this field combining with scientific papers about medical image fusion in recent years. This paper is mainly divided into the following sections:

(1) Introduction to the current fusion methods

(2) Mode of multimodal fusion

(3) Comparing the data of different medical image fusion methods in the same database with the same evaluation index

(4) Discussing the challenges of medical image fusion methods and future research trends

\section{Fusion Methods}

This chapter introduces the methods of medical image fusion from three aspects, the fusion method based on spatial domain, the fusion method based on transform domain, and the fusion method based on deep learning.

2.1. Spatial Domain. The medical image fusion technology based on spatial domain is the hot topic in early research. Its fusion technology is simple, and the fusion rules can be directly applied to the source image pixels to obtain the merged image. The fusion methods of spatial domain include the high-pass filtering method, the principal component analysis method, the saturation method of hue intensity, the average method, the maximum selection method, the minimum selection method, and the Brovey method. Due to the spectral distortion and spatial distortion in the fused image of the spatial domain, the heat of research in the spatial domain of the medical image fusion method is gradually decreasing in recent years. Researchers often use spatial domain fusion strategies as a part of the transformation domain to form new research methods.

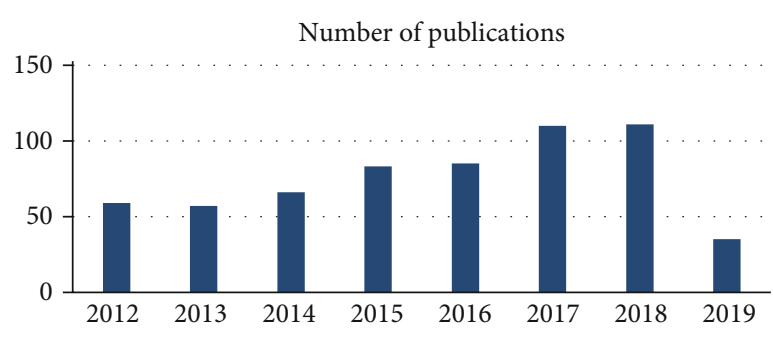

FIGURE 1: Number of published papers with of medical image fusion.

We will only briefly introduce the IHS method with a high usage value as below.

2.1.1. Fusion Method Based on IHS Domain. The IHS model proposed by an American scientist Munsell explains the characteristics of the human visual system. It has two characteristics: (1) the intensity component has nothing to do with the color information of the image; (2) hue and saturation components are closely related to the way people perceive color. Therefore, researchers often use this model to solve the color problem in the image fusion process, especially the fusion of PET/SPECT images with color information.

Chen [7] combined the IHS model with Log-Gabor transform to propose a new method about the fusion of MRI and PET and decomposed the PET image with IHS to obtain the three basic characteristics of hue $(\mathrm{H})$, saturation (S), and intensity (I). The component intensity represents the brightness of the image, so the intensity components of the MRI and PET images are decomposed by the LogGabor transform consisting of the logarithmic transformation of the Gabor filter to obtain the high-frequency subbands and the low-frequency subbands. Fusion of highfrequency subbands comes with maximum selection; fusion of low-frequency subbands comes with a new method based on two-level fusion of visibility measurement and weighted average rule. The inverse Log-Gabor transformed component and the original hue and saturation components are inversely HIS to obtain a fused image. It can effectively preserve the structures and details of the source image and reduce the color distortion. This method is superior to the existing IHS+FT method in visual perception. Haddadpour et al. [8] proposed a new fusion method which combining the IHS method with the two-dimensional Hilbert transform. The method [9] introduces the concept of BEMD when merging high- and low-frequency subbands. BEMD is called bidirectional empirical mode decomposition and is extended by empirical mode decomposition. It is widely used in biomedicine field because of its envelope surface. The algorithm has no obvious distortion and is superior to the PCA and wavelet algorithms in terms of contrast and color intensity. Its disadvantage is that the information entropy (EN) is relatively low. Figure 2 shows the framework of the IHS domain fusion method based on the fusion of MRI and PET images.

In order to achieve better results, different researchers tend to study the decomposition transform, such as DST and Log-Gabor transform. And they study fusion algorithm about the decomposition transform, such as SR algorithm and maximum selection algorithm. 


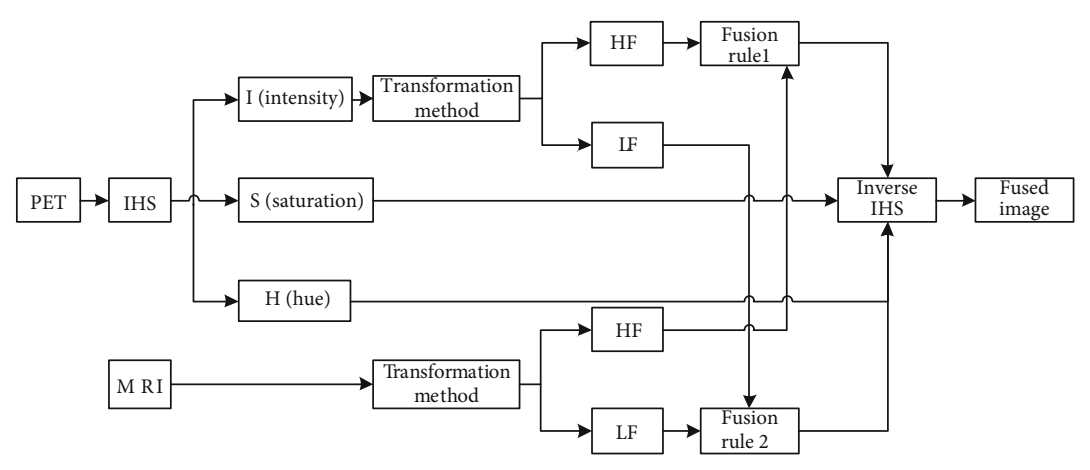

FIGURE 2: Framework diagram based on the IHS domain fusion method.

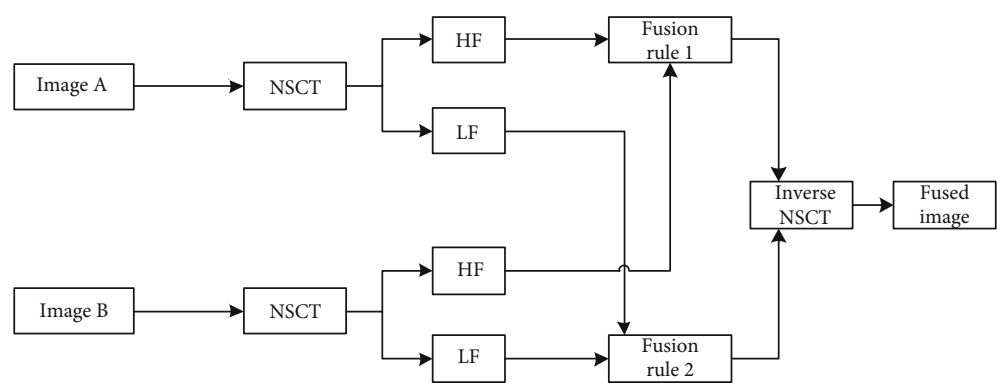

Figure 3: Framework diagram based on the NSCT domain fusion method.

2.2. Transform Domain. The medical image fusion methods of the transform domain are mostly based on the multiscale transform (MST) theory, and they are also the hotspots of research in recent years. The MST-based fusion method is generally divided into three steps: decomposition, fusion, and reconstruction. The medical image fusion method based on transform domain is transforming the source image from time domain to frequency domain or other domains to obtain the low-frequency coefficient and high-frequency coefficient. This section focuses on three most commonly used transformations in medical image fusion methods: nonsubsampled contourlet transform, nonsubsampled shearlet transform, and discrete wavelet transform.

\subsubsection{Fusion Based on Nonsubsampled Contourlet Transform} (NSCT). The contourlet transform is multiscale which is proposed by Do et al. [10]. It is suitable for constructing multiresolution and multidirectional situations and has advantages in smoothness processing. However, it does not have translation invariance, and it is easy to generate pseudo-Gibbs phenomenon (artifact) near the singular point of the reconstructed image, resulting in image distortion, so it is not the best choice for image fusion method. To this end, many researchers have done more in-depth research. After contourlet transform, Cunha et al. [11] proposed a multiscale decomposition method superior to contourlet transform, which is an improvement of contourlet transform, called nonsubsampled contourlet transform. NSCT has the characteristics of translation invariance and avoiding spectral aliasing. The structural information of the source image is preserved in the decomposition and reconstruction, and the direction information can be better extracted. Nonsub- sampled contourlet transform is one of methods widely used in medical image fusion of transform domain in recent years. Firstly, the source image is decomposed by NSCT to obtain the coarse layer and the detailed layer, and then, the multiscale and multidirection decompositions are calculated by NSPFB and NSDFB filters to obtain subband images with different scales and directions.

Coarse layer fusion with low-frequency band fusion rules:

$$
L_{k}^{F}(p)= \begin{cases}L_{K}^{A}(p), & \text { if } u_{K}^{A}(p) \geq u_{K}^{B}(p) \\ L_{K}^{B}(p), & \text { if } u_{K}^{A}(p)<u_{K}^{B}(p)\end{cases}
$$

Fusion detail layer with high-frequency band fusion rules:

$$
D_{k, h}^{F}(p)= \begin{cases}D_{K, h}^{A}(p), & \text { if } g_{K, h}^{A^{2}}(p) \geq g_{K, h}^{B^{2}}(p) \\ D_{K, h}^{B}(p), & \text { if } g_{K, h}^{A^{2}}(p)<g_{K, h}^{B^{2}}(p)\end{cases}
$$

Finally, the image is inverse NSCT to obtain a fused image. A block diagram of the NSCT-based fusion method is shown in Figure 3.

Most algorithms will do in-depth research and changes on the fusion rules.

The rules for merging high-frequency subbands are LogGabor, local energy-based weighting strategy, type-2 fuzzy logic algorithm [12], and adaptive two-channel pulsecoupled neural network algorithm (PCNN) [13], based on improved PCNN (IPCNN) [14] and significant matching measure rules [15]. 
The rules for merging low frequency subbands are phase consistency, weighting strategy based on gray mean deviation, local energy algorithm based on local features [12], sparse representation algorithm (SR) [13, 15], and based on improved PCNN (IPCNN) [14].

In order to solve quality problem of fused images, Xinqiang et al. [16] proposed an image fusion method based on local neighborhood features and NSCT. Firstly, NSCT processing is performed on the source image to obtain LF and $\mathrm{HF}$ in each direction, where a weighted fusion strategy based on gray mean deviation is used for LF, a weighted fusion strategy based on local energy is used for HF, and a fused image is transformed by inverse NSCT. In order to extract more useful feature information, Padmavathi et al. [17] proposed a new fusion method which combines Darwinian particle swarm optimization algorithm with NSCT. Elements in particle swarm optimization (PSO) can be used to extract the required features and remove redundant parts [18]. It is a good way to extract features. However, the shortcoming of PSO algorithm is that elements may be fixed on incorrect local optimal points. Darwinian particle swarm optimization algorithm is proposed to solve the shortcoming by DPO. The fusion image effect obtained by NSCT+DPSO algorithm is better than that of PSO, and the storage requirement is lower. Mohammed et al. [13] proposed a multimodal combination method which is based on the NCST, in which the sparse representation algorithm is used to fuse the lowfrequency band, and the high-frequency band is fused by the adaptive two-channel pulse-coupled neural network. The fusion image quality of this method is high and can be captured. The fusion image of this method has high quality, can capture subtle details, adapts to the characteristics of HVS, and shows good performance in both objective and subjective analysis. Because the method uses SR and PCNN algorithms, it causes large computational defects. Tian et al. [14] proposed an improved PCNN (IPCNN) multimodal medical image fusion algorithm based on the NSCT domain. In the traditional PCNN model, the local regional singular value was introduced as the connection strength parameter of the neurons in the PCNN model to construct the local structural information factor and activate the neurons to form the improved PCNN model. The model is used to fuse high- and low-frequency coefficients, and the fused image has better robustness, reliability, and visual effects. Recent research has emerged a fusion algorithm combining NSCTbased PCNN and shuffled frog leaping algorithm, which significantly improves the spatial resolution [19]. Shabanzade and Ghassemian [15] proposed that in the multimodal fusion method based on NSCT, the sparse representation algorithm is used to fuse the low-frequency bands, and how to select a good dictionary is the key to the sparse representation algorithm. Therefore, a dictionary learning algorithm based on the combination of principal component analysis and clustering method is proposed. It can effectively separate the significant characteristics of the low-pass band coefficient; overcome the shortcomings such as the slow speed of KSVD computer, the limitation of DCT basis, or wavelet basis by input image; and has the advantages of fast computing speed, low cost, compact structure, and strong adaptability.
At the same time, the high-frequency subband is fused by the explicit matching measure rule. This method is superior to the multiscale transform and sparse representation based on visual effects and quantitative indicators.

However, some researchers tend to combine NSCT and other algorithms into new methods.

Madanala and Jhansi Rani [6] combined the advantages of frequency and time localization of wavelet transform and displacement invariance of nonsubsampled contourlet transform and proposed a fusion framework based on DWC + NSCT domain cascade. In this framework, wavelet transform was used to decompose the source image in the first stage in order to obtain the detailed coefficient and approximate coefficient, and principal component analysis method was used to fuse the detailed coefficient and approximate coefficient to minimize the redundancy. Finally, inverse wavelet transform was used to obtain the reconstruction in the first stage. In the second stage, NSCT is applied to the products in the first stage to obtain the high-frequency and low-frequency coefficients. The maximum selection rule is used for fusion, and then, the final fusion image is obtained by inverse NSCT. The second stage solves the displacement variance problem generated in the first stage, which makes the fused image has the characteristics of strong applicability and good effect. Similarly, Bhateja et al. [20] cascaded the combination of stationary wavelet transform and nonsubsampled contour transform domain. This algorithm reduces the redundancy of fused images and enhances the contrast of diagnostic features.

2.2.2. Fusion Method Based on Nonsubsampled Shearlet Transform (NSST) Domain. In 2005, the tool shearlet proposed by Labate et al. [21] has multiscale, directional, and other characteristic but does not have translation invariance. Until 2007, Easley et al. [22] proposed a nonsubsampled shearlet transform, which solves the problem of translation invariance on the basis of retaining the directivity of shearlets. NSST consists of a Nonsubsampled Laplacian Pyramid (NSLP) and Multiple Shear Filters. The source image is decomposed into high-frequency components and lowfrequency components by NSLP, and then, the direction filter is used to process different subbands and coefficients in different directions, among which the low-frequency subband is iterative decomposition. Directional filtering is performed using a shear matrix, so it has a strong directivity. As shown in Figure 4, when the decomposition level is $m=3$, the image is decomposed into four subbands with $m+1=4$, the size of which is the same as that of the source image, thus ensuring the invariance of displacement [23]. Compared with NSCT, NSST has higher sensitivity and lower computational complexity, while overcoming the limitations of components with a certain number of directions.

2.2.3. Pulse-Coupled Neural Network (PCNN) Fusion Method Based on NSST Domain. NSST is a popular transformation that highlights feature information in medical image fusion. It is often favored by researchers because of its high sensitivity, multidirectionality, and high-speed processing capability. The pixel points generated by the NSST decomposition of the 


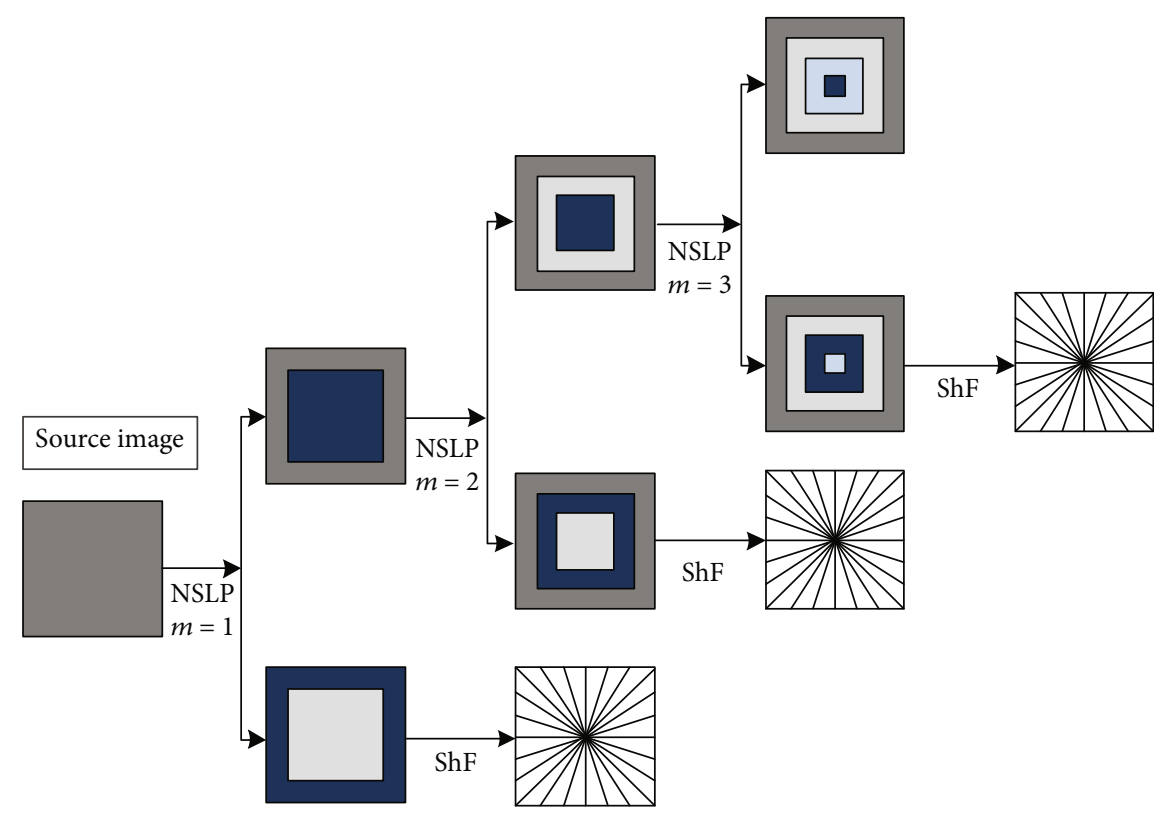

Figure 4: NSST diagram.

source image correspond to edge information and texture information with large transform coefficients, and the amount of multimodal medical image information acquired by different sensors is greatly different. The fusion image should not only keep the characteristic information of the source image but also to ensure good visual effect and less distortion, therefore to use PCNN fusion image decomposed high-frequency coefficient [24]. PCNN is a biologically inspired feedback neural network, which is a single-layer two-dimensional horizontally connected neuron array. The PCNN neurons are composed of a receiving field (dendritic), a connected modulation field, and a pulse generator. PCNN can be used to extract useful information from source images without training process, and the characteristics of neurons make it have greater advantage in biological background. It has been widely used in image processing field [25]. However, PCNN also has many defects such as too many parameters and difficulty in setting parameters. Therefore, researchers have proposed more optimization methods.

Yin et al. [26] proposed the PA-PCNN multimodal medical image fusion method based on NSST, which decomposed the multimodal source image NSST and obtained the multiscale and multidirection representation of the source image. A new fusion strategy is proposed to fuse the low-frequency coefficients. The activity-level metric defined as WLE in the strategy solves the energy preservation problem in the image fusion processing. In order to fully extract the details in the source image, a new activity level metric WSEML weighted sum was introduced. The parameter adaptive pulse-coupled neural network (PA-PCNN) model is used to fuse highfrequency coefficients, which solves the problem of difficult parameter setting in the traditional PCNN model. Finally, NSST reconstruction is performed. The algorithm has fast convergence speed, few iterations, and good effect. It is the first example applied to medical image fusion. Ouerghi et al. [27] proposed a simplified pulse-coupled neural network (S-PCNN) based on NSST. Unlike other fusion methods, this method converts PET images into YIQ components. The NSST transform only performed for MRI images and the Y components of PET images. The low-frequency subband is fused with the standard deviation of the weight region and the local energy. The high frequency is fused by the S-PCNN with the adaptive connection strength coefficient excitation. The fusion effect of this algorithm is better, but the application range is relatively narrow. There are still many researches on PCNN fusion methods based on NSST domain [28-30].

\subsubsection{Frei-Chen Operator Medical Image Fusion Method} Based on NSST Domain. Extracting the direction information of an image is a challenge of image fusion, and the Frei-Chen operator can obtain edge and direction information in the source image. The source image is scaled by the averaging filter to obtain 9 subgraphs, where W1-W4 is the edge subspace map, W5-W6 is the straight line, W7-W8 is the discrete Laplace transform, W5-W8 is the line subgraph, and W9 is the average of 9 subgraphs.

After Mishra et al. [31] used the Frei-Chen operator for infrared and visible image fusion to achieve good results, Ganasala [32] proposed a Frei-Chen operator medical image fusion method based on NSST domain. Similar to other methods, NSST decomposition is carried out on the source image, but in order to preserve the significance features and maintain the structural similarity of the source image, FreiChen operator is used to define the appropriate significance or activity measurement for the approximate subband and detail subband coefficients, and the coefficient is selected according to its measuring value. The quality standard values of image fusion in different data sets are better, and the quantitative evaluation indicators are superior to the existing methods. There are still many new algorithms based on the combination of the NSST domain and other algorithms [33, 34], which is still an area of interest for many researchers. 


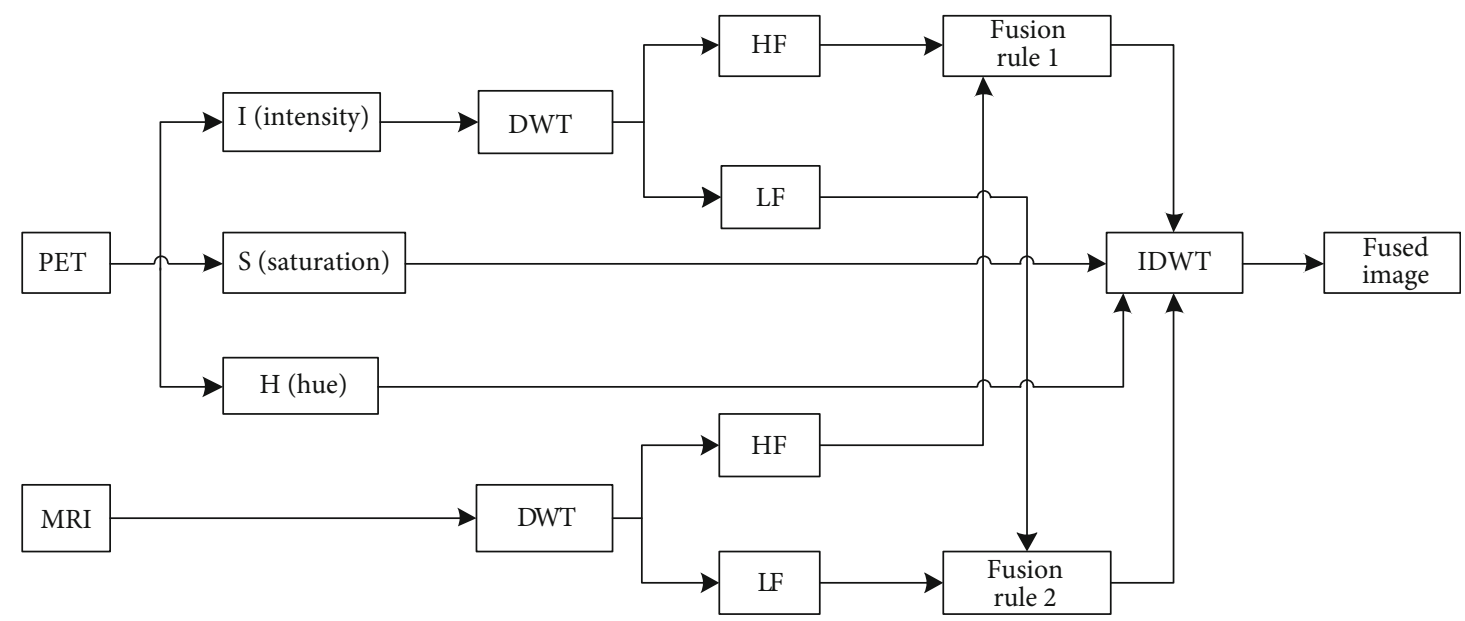

FIGURE 5: Block diagram based on the NSCT fusion method.

\subsubsection{Fusion Method Based on Discrete Wavelet Transform} (DWT). Discrete wavelet transform can make different input frequency signals maintaining stable output and has good positioning in the time domain and frequency domain, which helps to preserve the specific information of the image. Therefore, discrete wavelet transform (DWT) is the most widely used transform in the early research of multimodal medical image fusion algorithms. The discrete wavelet transform overcomes the limitations of the principle component analysis and has a good visual and quantitative fusion effect. Most of the DWT-based fusion methods are applied to MRI and PET image fusion $[35,36]$ but also to others [37]. The source image is preprocessed and enhanced, and the intensity component is extracted from the PET image using the IHS transform, which preserves more anatomical information and reduces color distortion. The DWT transform is performed on the intensity components of MRI and PET to obtain high- and lowfrequency subbands. The high- and low-frequency subbands are, respectively, fused by different fusion rules, and the inverse DWT transform is performed to obtain the fused image [38]. A block diagram based on the DWT fusion method is shown in Figure 5.

Most of the researchers have in-depth research on the fusion rules. Different fusion rules show different fusion effects. The fusion rule 1: the average method $[35,38]$; fusion rule 2 : fuzzy $-c$ means clustering [35]. In view of the shortcomings of discrete wavelet transform without displacement invariance and no phase information, the researchers introduced complex wavelet transform (CWT) [39]. On the basis of complex wavelet transform, Singh et al. [40] proposed a multimodal medical image fusion method based on Daubechies complex wavelet transform (DCxWT), which is superior to the spatial domain fusion method (PCA and linear fusion) and discrete wavelet method in transform domain. The dual-tree complex wavelet transform (DTCWT) proposed by Kingsbury [41] has directional selectivity and displacement invariance and can preserve the edge details of the source image. It is also an effective image fusion method $[42,43]$, but in image decomposition, the factors that are affected by the direction are relatively large. In recent years, researchers have often combined DTCWT with other algorithms to form new methods.
Padmavathi et al. [44] proposed a method based on the combination of dual-tree complex wavelet transform and principal component analysis (DTCWT-PCA). The principal component analysis method is one of the multivariate analysis methods based on eigenvectors. It is better to remove redundant information generated by DTCWT decomposition, and it is also the direction of block-level fusion development. Talbi and Kholladi [45] proposed a hybrid algorithm based on dual-tree complex wavelet transform and predator-prey optimizer (DTCWT+PPO), combining DTCWT and PPO, and using the mutual information technology to obtain the dual advantages of the two methods. The absolute high-value method is used to fuse the decomposed high-frequency coefficients, the weighted average method is used to fuse the low-frequency coefficients, the predator-optimizer is used to estimate and optimize the weights, and finally, the inverse transform is used to obtain the fused images. The algorithm is characterized by high robustness and high efficiency.

2.3. Image Fusion Based on Deep Learning. Deep learning is a new field of medical image fusion research in recent years. Convolutional neural network (CNN) is a typical deep learning model proposed by Krizhevsky et al. [46]. Compared with medical image fusion, deep learning is widely used in the segmentation of medical images [47-49] and registration of medical images [50-52]. The medical image fusion methods based on spatial domain and transform domain have the defects of activity level measurement (feature extraction) and fusion rules, which need artificial design, and the correlation between them is extremely small. In order to overcome the above problems, Liu et al. [53] applied CNN to image fusion for the first time in 2017, achieving good results relative to the spatial domain and the transform domain. The U-Net network model is widely used in medical image segmentation. From 2D to 3D [54,55], its research technology has been relatively mature and has achieved good results in the field of medical image segmentation, but medical image fusion is a new field.

$\mathrm{CNN}$ is a multistage feedforward artificial neural network with trainable supervised learning. The convolution 


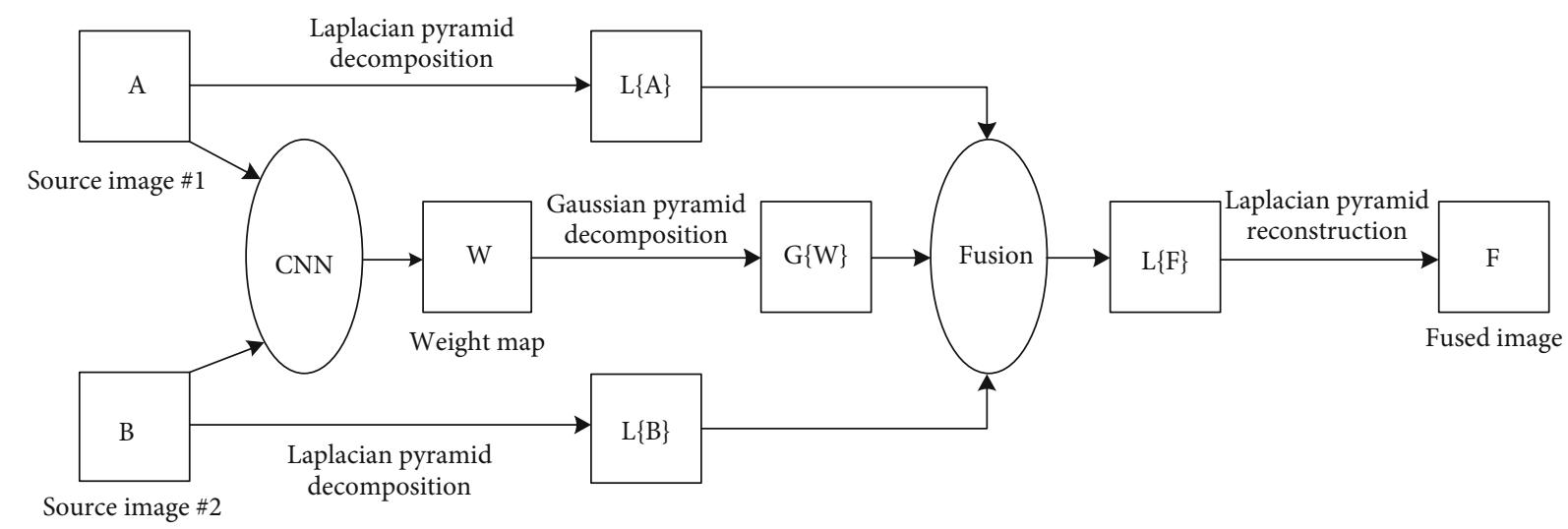

FIgURE 6: Schematic diagram based on CNN fusion algorithm.

operation is multidimensional. In a convolutional network, the first parameter is usually called an input, and the second parameter is called a kernel function, and the output is called a feature map. Sparse representations (also known as sparse weights), parameter sharing, and isomorphic representations are three important architectural ideas of CNN. Traditional neural networks use matrix multiplication to deal with connection relationships. An output unit is associated with each input unit, which inevitably requires a lot of storage. However, the nature of the sparse representation of the convolutional network and the neurons are only connected to several neurons adjacent to the previous stage, and the local convolution operation is performed, which reduces the storage requirements and improves the computational efficiency. CNN's parameter sharing abandons the nonuniqueness of weights in traditional networks. The weights in the $\mathrm{CNN}$ stage are constant, which is better than others in storage requirements. Traditional automatic encoders are fully connected. Vector output and source image are not necessarily aligned in space, while U-Net uses local connection structure. Vector output and source image are aligned in space, so the visual effect of fusion image is better. U-Net is a fullconvolution network [56], which consists of contraction path and expansion path. In-depth learning training needs a large number of samples, while U-Net is improved based on full convolution neural network, and can train a small number of samples using data enhancement. This advantage just caters to the shortcoming of a small sample size of medical image data.

\subsubsection{Image Fusion Method Based on Convolutional Neural} Network (CNN). Medical images differ in intensity at the same location, so the fusion method proposed in [53] is not suitable for medical image fusion. Yu et al. [57] first proposed a medical image fusion method based on CNN. This method uses the siamese network to generate weight map. The siamese network [58] is one of the three models for comparing patch similarity in the CNN model. Because its two weight branches are the same, the feature extraction or activity level measurement methods of the source image are the same. This has certain advantages over the models of pseudosiamese and 2-channel, and the ease of training of the siamese model is also the reason why it is favored in fusion applications. After obtaining the weight map, the Gaussian pyramid decomposition is used, and the pyramid transform is used for multiscale decomposition, so that the fusion process is more in line with human visual perception. In addition, the localized similarity-based fusion strategy is used to adaptively adjust the decomposed coefficients. The algorithm combines the common pyramid-based and similarity-based fusion algorithm with the $\mathrm{CNN}$ model to produce a superior fusion method. Figure 6 is a model of the algorithm.

$\mathrm{CNN}$ is a new challenge in the medical field; the main reasons are (a) a large number of annotated training set data is required, (b) training takes a long time, and (c) the convergence problem is complicated, and the overfitting needs to be adjusted repeatedly. As for the problem that a large number of annotated training sets are needed, Liang et al. [59] proposed that the MCFNet network method refers to different forms of medical image histograms and transforms 1.2 million natural images in ILSVRC 2013 ImageNet into medical images with similar intensity or texture distribution as training data sets. Reconstructed data sets are very similar to medical image data sets. In order to avoid overfitting, $256 * 256$ images are randomly extracted from the transformed images and trained with medical images. The optimization of the loss function of this method is still the direction of future research. Following Liu, Hermessi et al. [60] proposed the $\mathrm{CNN}+$ shearlet fusion method to achieve a good fusion effect. Using the full convolution siamese architecture, the training framework is the famous MatConvNet. It can well retain information, and visual perception is better than the CNN $+\mathrm{MF}$ method. However, there are some problems such as long training time and difficult architecture. This is also a direction for future research in this field. $\mathrm{Vu}$ et al. [61] proposed a fusion method based on the combination of sparse self-encoder and convolutional neural network. The preprocessing SAE was added to the CNN classifier, which is better than the previous CNN. The fusion method based on CNN began to develop [62-64].

2.3.2. Image Fusion Method Based on U-Net. The existing medical image fusion methods neglect the image semantics, do not pay attention to the processing of semantic conflicts, and lose useful semantic information. As a result, the fused image appears blurred boundary, which makes it more 
difficult for medical workers to parse the fused image. Fan et al. [65] proposed a medical image fusion method based on semantics, which solved the problem of semantic loss of fused images. In this algorithm, two U-Nets are used to construct the FW-Net network model. It is not the first time to combine U-Net with an automatic encoder in medical image research [66]. The left and right structures of FW-Net are the encoder and the decoder. They both follow the structure of U-Net. The encoder is used to extract the semantics of the source image, and the decoder is used to reconstruct the source image. FW-Net can extract the semantics of brightness in the source image and then automatically maps the brightness of different modal images to the same semantic space for image fusion. In order to obtain a smooth and clear image, bilinear interpolation is added to each layer of the encoder and decoder in FW-Net framework. There is no semantic conflict in the fused image, which is superior to other methods in visual effect. This algorithm is only applied to MRI and CT. Other pattern fusion, such as MR and PET fusion and MR and SPECT fusion, will be a research trend in the future. At the same time, the research of U-Net in medical image is not yet mature, so the research of U-Net in medical image fusion is also a focus.

\section{The Way of Multimodal Fusion}

MRI, also known as Magnetic Resonance Imaging, provides information on the soft tissue structure of the brain without functional information. The density of protons in the nervous system, fat, soft tissue, and articular cartilage lesions is large, so the image is particularly clear and does not produce artifacts. It has a high spatial resolution and no radiation damage to the human body, and the advantage of rich information makes it an important position in clinical diagnosis. The density of protons in the bone is very low, so the bone image of MRI is not clear. The CT image is called Computed Tomography imaging. The X-ray is used to scan the human body. The high-density absorption rate of bone tissue relative to soft tissue makes the bone tissue of the CT image particularly clear. The low permeability of X-rays in soft tissue leads to low absorption rate, so CT images show less cartilage information, which represents anatomical information. SPECT is called Single-Photon Emission Computed Tomography, which is a functional image that displays the metabolism of human tissues and organs and the blood flow of arteries and veins. It provides good and malignant information of tumors and is widely used in the diagnosis of various tumor diseases. However, the resolution of SPECT is low and the positioning ability is poor. The PET image is called Positron Emission Tomography, which reveals the true information of blood flow and can accurately identify the location of the patient's lesion. Its principle is using positrons to generate $\gamma$ photons in collision with electrons in the tissue. The purpose of PET is to detect the number of $\gamma$ photons, showing a color image of brain function information, suitable for tumor detection; its sensitivity is high, but it is difficult to obtain accurate brain structure position information; soft tissue and bone boundary resolution is lacking, so the spatial resolution is very low and the spatial distortion is highly probable.

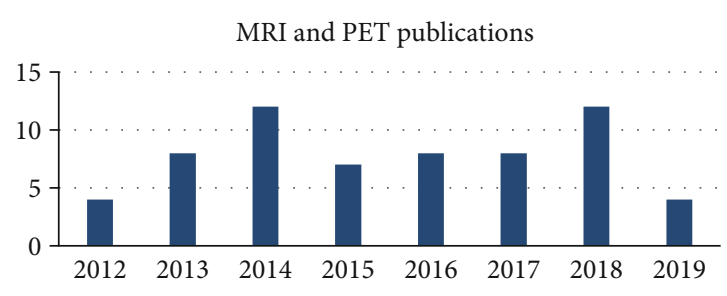

FIGURE 7: MRI/PET fusion research trend chart.

There are many fusions of imaging methods in medical image fusion, such as MRI and PET, MRI and CT, MRI and SPECT, CT and PET, CT and SPECT, SPECT and PET, and MRI-T1 and MRI-T2. Different ways of integration keep their own characteristics, such as MRI/PET fusion images which are important for detecting liver metastasis, Alzheimer's disease, and brain tumor diagnosis; MRI/SPECT fusion images are helpful for the localization of lesions and vertebral bone metastasis in tinnitus patients; CT/PET fusion image energy improves the diagnosis of lung cancer; SPECT/PET for abdominal research; and ultrasound/MRI for vascular blood flow diagnosis. The following will focus on a few hot ways of fusion.

3.1. MRI and PET Fusion. MRI is a gray image while PET is a color image, which is easily distorted in the fusion processing. In most fusion algorithms, the IHS model is used to decompose the intensity components of PET image [8], and BEMD, Log-Gabor transform, and other algorithms are combined to process these components, so as to preserve more color of PET image. Yin et al. [26] proposed an MRI and PET image fusion algorithm based on NSST and S_PCNN, which converts the PET image into YIQ component, and then used NSST to decompose MRI and the Y component of PET into low-frequency and high-frequency subbands. The simplified PCNN model was used to process highfrequency coefficients; the fused image has good effect, small color distortion, and rich structural information. Wang et al. [37] proposed a preparation method based on discrete wavelet transform for preprocessing of MRI and PET image fusion, which solved the problem of quality degradation and unreadability of input images, and the fusion accuracy was as high as $90 \%-95 \%$. Chaitanya et al. [67] proposed a new fusion method by combining shearlet transformation and discrete cosine transform. Arash and Javad [68] first applied adaptive filters to the fusion of MRI-PET images, using spatial and spectral difference criteria to optimize filter coefficients. There are other MRI/PET fusion methods [69-71]. MRI/PET images are often involved in the clinical diagnosis of Alzheimer's disease, and the fusion of MRI and PET images is what is needed to meet the diagnosis. MRI/PET is a key element in tumor diagnosis. In the near future, PET/MRI may emerge as a powerful multimodal technique in clinical oncology. Figure 7 shows the trend of articles related to MRI/PET fusion research in recent years (statistical time is from 2012 to August 2019).

3.2. MRI and CT Fusion. The combination of MRI and CT combines the advantages of clear bone information in CT 


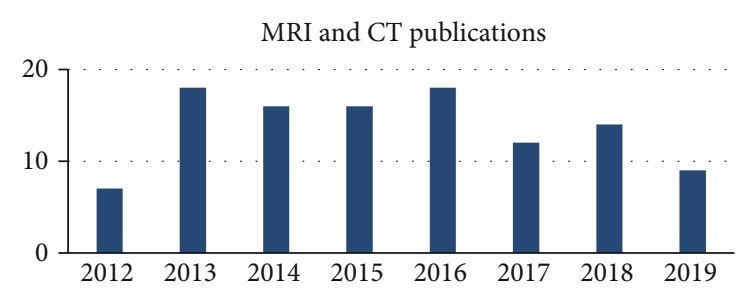

FIGURE 8: MRI/CT fusion research trend chart.

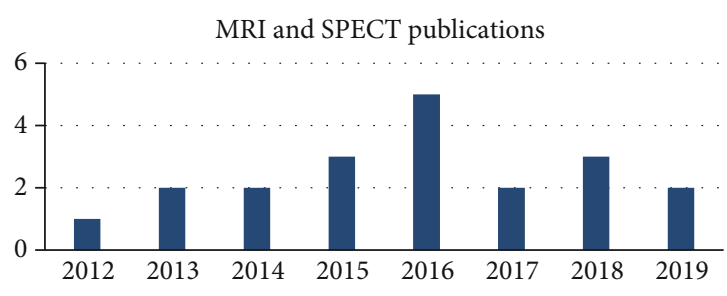

FIGURE 9: MRI/SPECT fusion study trend chart. images and the clear soft tissue of MRI images to compensate for the lack of information in a single imaging. $\mathrm{Na}$ et al. [72] proposed a MRI and CT fusion algorithm based on guided filtering (GF). The fused image not only preserves the edge information of the source image but also extracts the feature information, which solves the problem of edge degree and clarity. In [31], the Frei-Chen operator fusion algorithm based on NSST domain is proposed. The visual analysis of fusion results has obvious improvement in contrast and structural similarity. Quantitative evaluation is also a further improvement of existing methods. In [73], the membership degree is difficult to select based on the intuitionistic fuzzy inference fusion algorithm. Mishra et al. [31] further proposed the fuzzy-PCNN rule, using multiple membership functions to generate fuzzy membership from specific parts of high-frequency coefficients; L2 norm set operation is applied to the results, using the rule to fuse the highfrequency coefficient; SF, EN, and SD of fused image have a higher value. Following [74] fusion of MRI/CT images in the NSST domain, Singh et al. [33] proposed a new fusion method using the ripple transform and NSST transform cascade, which has a good effect on visual quality and quantitative indicators. Other methods for MRI/CT image fusion include contourlet transform based on non-sub-sampling [75] and multiscale and multiresolution methods [76]. Figure 8 shows the trend of articles related to MRI/CT fusion studies in recent years (the statistical time is from 2012 to August 2019).

3.3. MRI and SPECT Fusion. The fusion image of MRI and SPECT has both functional information and structural information, which greatly helps noninvasive diagnosis. In order to extract the salient features of the image, Shahdoosti and Tabatabaei [77] proposed a new fusion algorithm by combining the antolony algorithm with the integrated empirical mode decomposition domain (EEMD), which provides a lot of spatial and color information. Du et al. [78] proposed the fusion method of anatomical image and functional image fusion using parallel significant features. Different significant features were utilized in image fusion such as MRICBV/SPECT-Tc and MRI-T1/SPECT-FDG. Fusion images could retain edge information in anatomical image and color detail information in functional image, with advantages of high spatial resolution and high intensity. In the fusion method based on NSST domain proposed by Xiong et al. [25], PA-PCNN and the new fusion strategy were used to fuse the high- and low-frequency subbands. The algorithm is superior to NSCT+SF+PCNN, SR+SOMP, GF, and NSCT+PCPD in terms of color preservation, and it is better than the NSCT+RPCNN method in detail preservation. It is an effective MRI/SPECT image fusion method. Daniel [79] proposed a homomorphic wavelet fusion method based on hybrid genetic-gray optimization algorithm, which is superior to DCTDWT FFT in mutual information, semaphore, and edge information. A fixed proportion of segmentation is prone to color distortion; Jing et al. [80] in this paper proposes a new adaptive decomposition algorithm for this problem to distinguish between low frequency and high frequency which can keep color and structural information; the algorithm will be sparse representation (SR), and the edge filter is applied to image fusion, in the aspect of color information and spatial information retained superior to other methods. Figure 9 shows the trend of articles related to MRI/SPECT fusion studies in recent years (statistical time is from 2012 to August 2019).

\section{Comparative Analysis}

4.1. Experimental Data Set. Most of the experimental images of medical image fusion come from the whole brain Atlas database (http://www.med.harvard.edu/aanlib/home.html). The whole brain Atlas is a benchmark database for evaluating the performance of multimodal medical image fusion methods developed by Keith A. Johnson of Harvard Medical School and MIT Journal Alex Becker. The database contains various data such as normal brain, cerebrovascular disease, neoplastic disease, recessive disease, inflammatory disease, or infectious disease. A few original images are also can be found at http://www.bic.mni.mcgill.ca/brainweb/.

4.2. Performance Analysis. Image fusion quality needs to be measured by a consistently accepted standard. These objective evaluation indicators are EN (entropy), MI (mutual information), standard deviation (SD), peak signal to noise ratio (PSNR), structural similarity index measure(SSIM), average gradient $(\mathrm{AG}), Q^{A B / F}$ metric, root mean square error (RMSE), edge intensity (ES), visual information fidelity (VIF), spatial frequency (SF), etc. A few common evaluation indicators are as follows.

4.2.1. Entropy (EN). Entropy describes the content of the information in the image. It is a measure of the amount of information contained in an image, taking values between 0 and 8 . The formula is as follows:

$$
\mathrm{EN}=-\sum_{L=0}^{L-1} p_{i} \times \log _{2} p_{i}
$$


where $L$ represents the number of gray levels and is a probability density function for each gray value $i$. The entropy value is proportional to the amount of information contained in the fused image.

4.2.2. Standard Deviation (STD). The standard deviation is mainly used to measure the overall contrast of the fused image and is used to determine the difference between the data and the average. If the STD value is larger, the more useful information the fused image contains, the better the fusion effect performs, and the image is clearer. The STD calculation formula is as follows:

$$
\mathrm{STD}=\sqrt{\frac{\sum_{i=1}^{M} \sum_{j=1}^{N}(f(i, j)-\mu)^{2}}{M N}},
$$

where $M$ and $N$ represent the length and width of the image $f(i, j)$, which is generally 256 . The average value of the fused image is represented by $\mu$.

4.2.3. Mutual Information (MI). Mutual information is a measure of the dependence between two input source images $(X, Y)$. Mutual information is how much information is calculated in the source image and transferred to the fused image. The mutual information is proportional to the fused message. The formula for calculating MI is as follows:

$$
\begin{aligned}
\mathrm{MI} & =I(x, f)+I(y, f), \\
I(x, y) & =\sum_{y \in Y} \sum_{x \in Y} p(x, y) \log \frac{p(x, y)}{p(x) p(y)},
\end{aligned}
$$

where $p(x)$ and $p(y)$ are the edge probability density functions of the two images, and $p(x, y)$ is the joint probability density function of the fused image and the source image $X, Y$.

4.2.4. Peak Signal to Noise Ratio (PSNR). PSNR is a quantitative measurement method based on mean square error. In the fusion image, the higher the PSNR is, the better the $\mathrm{SNR}$ is, and the closer it is to the source image.

$$
\operatorname{PSNR}=10 * \log _{10}\left(\frac{L^{2}}{\mathrm{RMSE}^{2}}\right)
$$

It represents the maximum pixel gray value in the fused image, which is generally 255. RMSE is the mean square error, and its calculation formula is

$$
\operatorname{RMSE}=\sqrt{\frac{\sum_{m=1}^{M} \sum_{n=1}^{N}[\operatorname{ground}(m, n)-\operatorname{fused}(m, n)]^{2}}{M \times N}} .
$$

Mean square error is an image quality measurement method. The value of RMSE is inversely proportional to the quality. The lower the value of RMSE, the better the quality of the fused image have. $\operatorname{ground}(m, n)$ and $\operatorname{fused}(m, n)$ represent the intensity values of the source image and the fused image pixel, respectively, and the length and width of the image are $M$ and $N$, respectively.

4.2.5. Structural Similarity Index Measure (SSIM). SSIM is a measure of the structural similarity between a fused image and a source image. Its value is between 0 and 1, with 0 indicating zero correlation with the original image and 1 indicating the exact same image. The larger the SSIM value, the more similar the fused image is to the source image; that is, the fusion effect is better.

$$
\operatorname{SSIM}_{(A, B, F)}=0.5 \times\left(\operatorname{SSIM}_{(A, F)}+\operatorname{SSIM}_{(B, F)}\right)
$$

In

$$
\begin{aligned}
\operatorname{SSIM}_{(A, F)} & =\frac{\left(2 \mu_{A} \mu_{F}+C_{1}\right)\left(2 \sigma_{A F}+C_{2}\right)}{\left(\mu_{A}^{2}+\mu_{F}^{2}+C_{1}\right)\left(\sigma_{A}^{2}+\sigma_{F}^{2}+C_{2}\right)}, \\
\operatorname{SSIM}_{(B, F)} & =\frac{\left(2 \mu_{B} \mu_{F}+C_{1}\right)\left(2 \sigma_{B F}+C_{2}\right)}{\left(\mu_{B}^{2}+\mu_{F}^{2}+C_{1}\right)\left(\sigma_{B}^{2}+\sigma_{F}^{2}+C_{2}\right)} .
\end{aligned}
$$

$\mu_{A}, \mu_{B}$, and $\mu_{F}$ are the average values of the source image and the fused image, respectively; $\sigma_{A}^{2}, \sigma_{B}^{2}$, and $\sigma_{F}^{2}$ are the variances of the source image and the fused image, respectively; $\sigma_{A F}$ and $\sigma_{B F}$ represent the joint variance of the two source images and the fused image, respectively.

4.2.6. Spatial Frequency (SF). The spatial frequency reflects the sharpness of the fused image, that is, the rate of change of the image gray; the larger the SF is, the higher the image resolution perform.

$$
\mathrm{SF}=\sqrt{\mathrm{RF}^{2}+C F^{2}}
$$

In

$$
\begin{aligned}
& \mathrm{RF}=\sqrt{\frac{1}{M(N-1)} \sum_{i=1}^{M} \sum_{j=2}^{N}(X(i, j-1)-X(i, j))^{2}}, \\
& \mathrm{CF}=\sqrt{\frac{1}{(M-1) N} \sum_{i=2}^{M} \sum_{j=1}^{N}(X(i, j)-X(i-1, j))^{2}} .
\end{aligned}
$$

$\mathrm{RF}$ and CF are the row and column frequencies of the image, respectively.

4.2.7. $Q^{A B / F}$ Measurement. $Q^{A B / F}$ measures the amount of edge information from the source image to the fused image through the Sobel edge detection operator. The larger the value of $Q^{A B / F}$ represent, the more information is converted from the source image, and the edge information is better preserved. In general, high edge strength has a greater impact on $Q^{A B / F}$ than low edge strength.

$$
Q^{A B / F}=\frac{\sum_{n=1}^{N} \sum_{m=1}^{M}\left(Q^{A}(n, m) W^{A}(n, m)+Q^{B}(n, m) W^{B}(n, m)\right)}{\sum_{n=1}^{N} \sum_{m=1}^{M}\left(W^{A}(i, j)+W^{B}(i, j)\right)},
$$



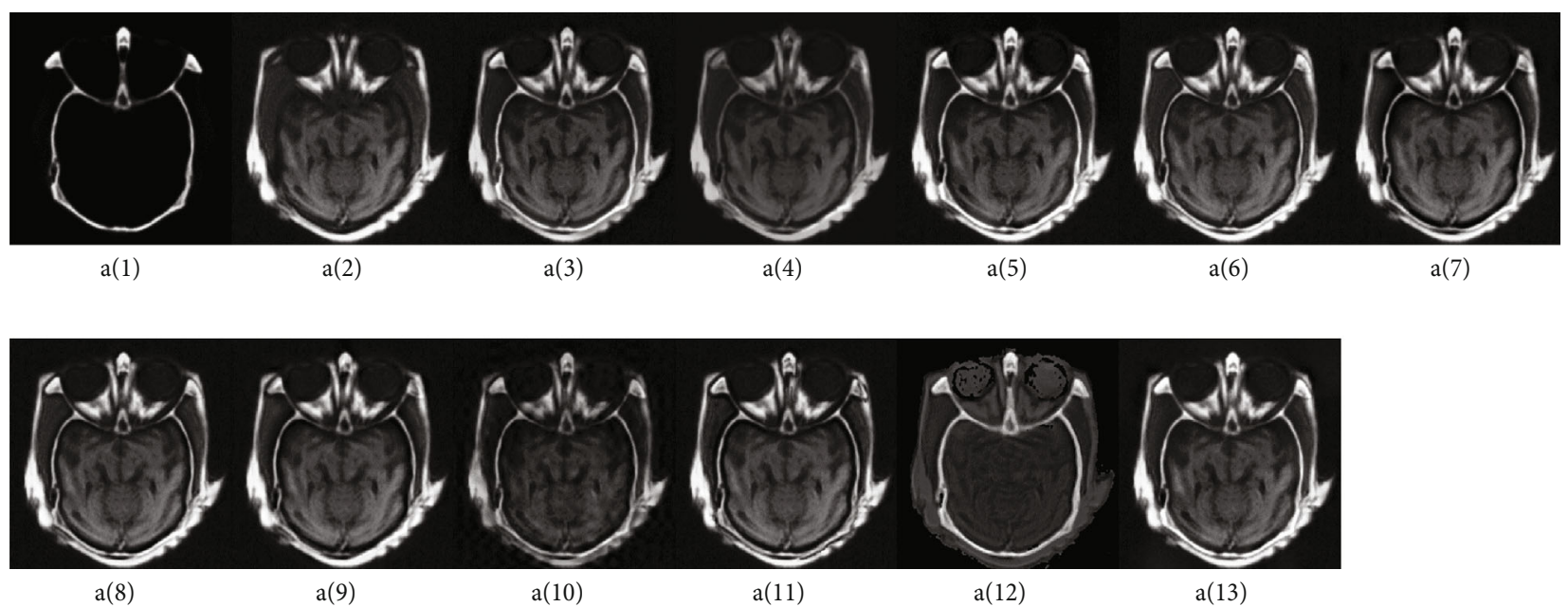

FIGURE 10: Examples of MRI-CT medical image fusion, a(1) and a(2) are CT and MRI source images, respectively. a(3) a(13) are the fused images of GFF [81], MSA [82], NSCT+SR [83], NSCT+PCNN [84], NSCT+LE [85], NSCT+RPCNN [86], NSST+PAPCNN [26], DWT [87], DWT+WA [88], U-Net [65], and CNN [57], respectively.

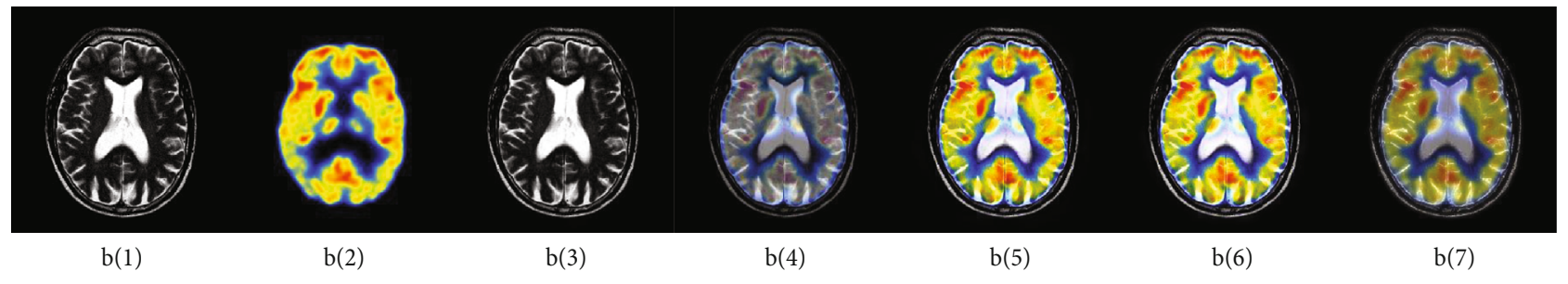

Figure 11: Examples of MRI-PET medical image fusion, $\mathrm{b}(1)$ and $\mathrm{b}(2)$ are MRI and PET source images, respectively. $\mathrm{b}(3) \sim \mathrm{b}(7)$ are the fused images of GFF [81], MSA [82], NSCT+LE [85], NSST+PAPCNN [26], and ESF+CSF [78], respectively.

where $Q^{A}(n, m), Q^{B}(n, m)$ is the edge information storage value; $W^{A}(n, m), W^{B}(n, m)$ is the weighting map.

4.3. Experiments and Analyses. To compare the three classifications of the medical image fusion methods in Section 2, we performed two experiments: MRI/CT and MRI/PET. The resolution of each test image is set to $256 \times 256$. The images are shown in Figures 10 and 11; in Figure 10, a(1) and a(2) show CT and MRI source images, respectively, and in Figure $11, b(1)$ and $b(2)$ show MRI and PET source images, respectively. Medical image pairs are obtained from http:// www.med.harvard.edu/aanlib/home.html.

4.3.1. Experiment and Evaluation on the Group of MRI-CT Fusion. In this section, the methods for comparison are eleven MRI-CT image fusion algorithms based on GFF [81], MSA [82], NSCT+SR [83], NSCT+PCNN [84], NSCT $+\mathrm{LE}$ [85], NSCT+RPCNN [86], NSST+PAPCNN [26], DWT [87], DWT+WA [88], U-Net [65], and CNN [57], respectively. From a(3) to a(13) in Figure 10 show the fused images of these fusion methods. In order to evaluate the performance of the above different multimodal medical image fusion methods, seven metrics are applied to the objective quality assessments, such as EN, STD, MI, PSNK, SSIM, SF, and $Q^{A B / F}$ [89]. After that, the objective performances of those methods are shown in Table 1 (the highest value of each metrics is marked in underline).

From Table 1, the highest $Q^{A B / F}$ value is the algorithm GFF, which saves the edge information of the source image better. The MSA algorithm and MSCT+SR performed best on the PSNK and STD evaluation indicators, respectively. The NSCT+PCNN algorithm performs well on the MI and SSIM evaluation indicators. The fusion image $\mathrm{a}(6)$ is also superior to other fusion methods in terms of visual effects, indicating that the fusion image has more useful information and is similar to the source image. The sharpness of $\mathrm{a}(11)$ in Figure 10 is better than other methods, so the algorithm DWT + WA has the highest value on SF. The advantage of deep learning lies in feature extraction, so the algorithm CNN achieves the best in the EN index, and the amount of information contained in the fused image is the largest.

4.3.2. Experiment and Evaluation on the Group of MRI-PET Fusion. In this section, the methods for comparison are eleven MRI-PET image fusion algorithms based on GFF [81], MSA [82], NSCT+LE [85], NSST+PAPCNN [26], and ESF+CSF [78], respectively. $b(3)$ to $b(7)$ in Figure 11 show the fused images of these fusion methods. In order to evaluate the performance of the above different multimodal medical image fusion methods, seven metrics are applied to the 
TABLE 1: Evaluation index data of different methods of MRI-CT.

\begin{tabular}{lcccccccc}
\hline & \multirow{2}{*}{ Fusion methods } & & & \multicolumn{2}{c}{ MRI-CT evaluation standards } & \\
& & EN & STD & MI & PSNR & SSIM & SF & $Q^{A B / F}$ \\
\hline \multirow{2}{*}{ Spatial domain } & GFF [81] & 6.7971 & 53.7262 & 3.4313 & 31.1594 & 0.4865 & 16.0034 & $\underline{0.785}$ \\
& MSA [82] & 6.3804 & 43.5218 & 3.9185 & $\underline{35.0709}$ & 0.4829 & 10.8225 & 0.5526 \\
\hline \multirow{4}{*}{ Transform domain } & NSCT+SR [83] & 6.8938 & $\underline{60.7771}$ & 3.127 & 29.5602 & 0.4825 & 17.6378 & 0.7761 \\
& NSCT+PCNN [84] & 6.7391 & 58.8135 & $\underline{5.0068}$ & 31.2341 & $\underline{0.5043}$ & 17.0262 & 0.7775 \\
& NSCT+LE [85] & 6.8011 & 57.3437 & 3.2865 & 31.6099 & 0.4861 & 16.9265 & 0.7369 \\
& NSCT+RPCNN [86] & 6.7696 & 57.972 & 4.4187 & 31.6844 & 0.5002 & 17.2528 & 0.7659 \\
& NSST+PAPCNN [26] & 6.8406 & 57.2625 & 3.2309 & 32.9194 & 0.4914 & 15.8548 & 0.7059 \\
& DWT [87] & 6.5888 & 41.9861 & 1.9772 & 31.972 & 0.4293 & 13.3888 & 0.5507 \\
& DWT+WA [88] & 6.2981 & 55.4057 & 4.7547 & 30.9814 & 0.4875 & $\underline{18.1062}$ & 0.7772 \\
\hline \multirow{2}{*}{ DL } & U-Net [65] & 5.13 & 42.8826 & 2.2207 & 26.4196 & 0.3225 & 17.7593 & 0.3127 \\
& CNN [57] & $\underline{7.0062}$ & 60.0385 & 3.0757 & 28.9646 & 0.4751 & 17.6211 & 0.7747 \\
\hline
\end{tabular}

TABLE 2: Evaluation index data of different methods of MRI-PET.

\begin{tabular}{lcccccrrrr}
\hline & \multirow{2}{*}{ Fusion methods } & & \multicolumn{4}{c}{ MRI-PEG evaluation standards } \\
& & EN & STD & MI & PSNR & SSIM & SF & $Q^{A B / F}$ \\
\hline \multirow{2}{*}{ Spatial domain } & GFF [81] & 4.4312 & 63.882 & $\underline{3.127}$ & $\underline{42.9419}$ & $\underline{0.7098}$ & $\underline{31.7488}$ & $\underline{0.5978}$ \\
& MSA [82] & 4.5174 & 55.9475 & 2.8495 & 28.75 & 0.662 & 17.0158 & 0.3038 \\
\hline \multirow{2}{*}{ Transform domain } & NSCT+LE [85] & 4.5659 & 75.5464 & 2.6052 & 26.0083 & 0.6729 & 31.337 & 0.5184 \\
& NSST+PAPCNN [26] & 4.62 & $\underline{77.6748}$ & 2.6297 & 25.2629 & 0.673 & 31.7002 & 0.5206 \\
\hline Others & ESF+CSF [78] & $\underline{4.907}$ & 60.3976 & 2.84 & 29.6479 & 0.6483 & 21.0867 & 0.2845 \\
\hline
\end{tabular}

objective quality assessments, such as EN, STD, MI, PSNK, SSIM, SF, and $Q^{A B / F}$ [89]. After that, the objective performances of those methods are shown in Table 2 (the highest value of each metrics is marked in underline).

From Table 2, the GFF algorithm performs well on the MI, PSNR, SSIM, SF, and $Q^{A B / F}$ evaluation indicators. This algorithm uses guided filtering to control the saliency and spatial consistency of pixels, thereby improving the quality of the fused image. The highest STD value is the algorithm NSST+PAPCNN. Firstly, this algorithm converts PET color images to YUV, then, fuses the Y component with MRI. It can maintain the color without distortion, and the fusion effect is very good.

\section{Summary}

The development of medical image fusion ranges from spatial domain, transform domain, to deep learning. Its rapid development also indicates a high demand of computeraided clinical diagnosis. Different researchers propose different fusion methods, each of which has its own advantages in different evaluation indicators. However, there are nearly 30 kinds of evaluation indexes for medical image fusion. The quantitative evaluation indicators used by researchers are often different for different fusion effects. The nonuniqueness of evaluation indicators brings limitations to the application prospects. On the other hand, although the research on medical image fusion is very popular, its innovation is relatively low. Most of the fusion methods are modified based on the original methods, and the problems existing in the fusion effect are only improved but not completely solved, such as color distortion and feature information extraction. Applying innovative algorithms to medical image fusion remains a huge challenge in this area of research. Deep learning has improved the effect of fusion, but the research also has certain defects; for example, the framework of deep learning is single and the amount of data for training is small. Because the trained images require professional labeling by medical experts, the workload is large and the cost is high. Therefore, the training data is lacking, and the lack of data may lead to overfitting. Applying the feature information and the lesion information to the data set obtained by the data augmentation has a certain influence on the accuracy. Therefore, how to obtain a huge data set is a difficult point in medical image research. The training of deep learning is time-consuming, and the framework is complex, which requires high requirements for computer hardware configuration. It is an important part of its research to simplify the training model or put forward a new training model and parallel training. Partial fusion method relies on accurate image registration and has little independence.

There is a difference in medical image information obtained by different sensors. The current research hotspot is the fusion of two modes, and the fusion of the three modes is rarely studied. The two modal studies focus on the fusion of MRI/CT, MRI/PET, and MRI/SPECT. From the 
experiments in Tables 1 and 2, we can see that the most open source is MRI/CT, so researchers favor the research of MRI/CT fusion methods; other fusions are still a challenge. Some fusion algorithms are only for single fusion methods such as MRI/CT or MRI/PET, and the compatibility of the algorithm is relatively small. Clinical diagnostic needs are not limited to the fusion of structural and functional information images, such as thyroid tumor diagnosis needs CT, MRI, SPECT, and B-ultrasound; future fusion of multiple modes and algorithm compatibility is a challenging topic.

To sum up, this article from the medical image fusion method and different image fusion methods on medical image fusion research in recent years are discussed, combining the proposed fusion method in recent years and the advantages of different methods and fusion effect; for the way the different imaging fusion method and the research trend of statistics, this paper expounds the platform of research and data sets. According to the previous part, the research of deep learning in medical image fusion is the future trend.

\section{Disclosure}

We the undersigned declare that this manuscript entitled "A Review of Multimodal Medical Image Fusion Techniques" is original, has not been published before, and is not currently being considered for publication elsewhere.

\section{Conflicts of Interest}

The authors declare that they have no conflicts of interest.

\section{Acknowledgments}

This work was partly supported by the National Natural Science Foundation of China (NSFC) (Grant Nos.: 61861004 and 61762007) and the Natural Science Foundation of Guangxi, China (Grant Nos.: 2017GXNSFAA198267 and 2017GXNSFAA198269).

\section{References}

[1] B. Meher, S. Agrawal, R. Panda, and A. Abraham, "A survey on region based image fusion methods," Information Fusion, vol. 48, pp. 119-132, 2019.

[2] A. P. James and B. V. Dasarathy, "Medical image fusion: a survey of the state of the art," Information Fusion, vol. 19, pp. 419, 2014.

[3] P. H. Venkatrao and S. S. Damodar, "HWFusion: holoentropy and SP-whale optimisation-based fusion model for magnetic resonance imaging multimodal image fusion," IET Image Processing, vol. 12, no. 4, pp. 572-581, 2018.

[4] W. Zhao and H. Lu, "Medical image fusion and denoising with alternating sequential filter and adaptive fractional order total variation," IEEE Transactions on Instrumentation and Measurement, vol. 66, no. 9, pp. 2283-2294, 2017.

[5] N. Iqbal, S. Saleem, W. S. Jehan, and K. Ahmad, "Reduction of speckle noise in medical images using stationary wavelet transform and fuzzy logic," in 2017 International Symposium on
Recent Advances in Electrical Engineering (RAEE), Islamabad, Pakistan, October 2017.

[6] S. Madanala and K. Jhansi Rani, "PCA-DWT based medical image fusion using non sub-sampled contourlet transform," in 2016 International Conference on Signal Processing, Communication, Power and Embedded System (SCOPES), Paralakhemundi, India, October 2016.

[7] C.-I. Chen, "Fusion of PET and MR brain images based on IHS and Log-Gabor transforms," IEEE Sensors Journal, vol. 17, no. 21, pp. 6995-7010, 2017.

[8] M. Haddadpour, S. Daneshvar, and H. Seyedarabi, "PET and MRI image fusion based on combination of 2-D Hilbert transform and IHS method," Biomedical Journal, vol. 40, no. 4, pp. 219-225, 2017.

[9] M. S. Dilmaghani, S. Daneshvar, and M. Dousty, "A new MRI and PET image fusion algorithm based on BEMD and IHS methods," in 2017 Iranian Conference on Electrical Engineering (ICEE), Tehran, Iran, May 2017.

[10] M. N. Do and M. Vetterli, "The contourlet transform: an efficient directional multiresolution image representation," IEEE Transactions on Image Processing, vol. 14, no. 12, pp. 20912106, 2005.

[11] A. L. da Cunha, J. Zhou, and M. N. Do, “The nonsubsampled contourlet transform: theory, design, and applications," IEEE Transactions on Image Processing, vol. 15, no. 10, pp. 30893101, 2006.

[12] Y. Yang, Y. Que, S. Huang, and P. Lin, "Multimodal sensor medical image fusion based on type-2 fuzzy logic in NSCT domain," IEEE Sensors Journal, vol. 16, no. 10, pp. 3735$3745,2016$.

[13] A. Mohammed, K. L. Nisha, and P. S. Sathidevi, "A novel medical image fusion scheme employing sparse representation and dual PCNN in the NSCT domain," in 2016 IEEE Region 10 Conference (TENCON), Singapore, Singapore, November 2016.

[14] Y. Tian, Y. Li, and F. Ye, "Multimodal medical image fusion based on nonsubsampled contourlet transform using improved PCNN," in 2016 IEEE 13th International Conference on Signal Processing (ICSP), pp. 799-804, Chengdu, China, November 2016.

[15] F. Shabanzade and H. Ghassemian, "Multimodal image fusion via sparse representation and clustering-based dictionary learning algorithm in nonsubsampled contourlet domain," in 2016 8th International Symposium on Telecommunications (IST), Tehran, Iran, September 2016.

[16] Q. Xinqiang, Z. Jiaoyue, and H. Gang, "Image fusion method based on the local neighborhood feature and nonsubsampled contourlet transform," in 2017 2nd International Conference on Image, Vision and Computing (ICIVC), pp. 396-400, Chengdu, China, June 2017.

[17] Mahima, N. B. Padmavathi, and M. V. Karki, "Feature extraction using DPSO for medical image fusion based on NSCT," in 2017 2nd IEEE International Conference on Recent Trends in Electronics, Information \& Communication Technology (RTEICT), pp. 265-269, Bangalore, India, May 2017.

[18] H. H. Inbarani, A. T. Azar, and G. Jothi, "Supervised hybrid feature selection based on PSO and rough sets for medical diagnosis," Computer Methods and Programs in Biomedicine, vol. 113, no. 1, pp. 175-185, 2014.

[19] C. Huang, G. Tian, Y. Lan et al., “A new pulse coupled neural network (PCNN) for brain medical image fusion empowered 
by shuffled frog leaping algorithm," Frontiers in Neuroscience, vol. 13, 2019.

[20] V. Bhateja, H. Patel, A. Krishn, A. Sahu, and A. Lay-Ekuakille, "Multimodal medical image sensor fusion framework using cascade of wavelet and contourlet transform domains," IEEE Sensors Journal, vol. 15, no. 12, pp. 6783-6790, 2015.

[21] D. Labate, W.-Q. Lim, G. Kutyniok, and G. Weiss, "Sparse multidimensional representation using shearlets," in Wavelets XI, pp. 254-262, San Diego, California, USA, August 2005.

[22] G. Easley, D. Labate, and W. Q. Lim, "Sparse directional image representations using the discrete shearlet transform," Applied and Computational Harmonic Analysis, vol. 25, no. 1, pp. 2546, 2008.

[23] S. Singh, D. Gupta, R. S. Anand, and V. Kumar, "Nonsubsampled shearlet based CT and MR medical image fusion using biologically inspired spiking neural network," Biomedical Signal Processing and Control, vol. 18, pp. 91-101, 2015.

[24] R. Eckhorn, H. Journal Reitboeck, M. Arndt, and P. Dicke, "A neural network for feature linking via synchronous activity," Canadian Journal of Microbiology, vol. 46, no. 8, pp. 759763, 1989.

[25] Y. Xiong, Y. Wu, Y. Wang, and Y. Wang, "A medical image fusion method based on SIST and adaptive PCNN," in 2017 29th Chinese Control And Decision Conference (CCDC), pp. 5189-5194, Chongqing, China, May 2017.

[26] M. Yin, X. Liu, Y. Liu, and X. Chen, "Medical image fusion with parameter-adaptive pulse coupled neural network in nonsubsampled shearlet transform domain," IEEE Transactions on Instrumentation and Measurement, vol. 68, no. 1, pp. 49-64, 2019.

[27] H. Ouerghi, O. Mourali, and E. Zagrouba, "Non-subsampled shearlet transform based MRI and PET brain image fusion using simplified pulse coupled neural network and weight local features in YIQ colour space," IET Image Processing, vol. 12, no. 10, pp. 1873-1880, 2018.

[28] N. S. Mishra and S. Dhabal, "On combination of fuzzy memberships for medical image fusion using NSST based fuzzyPCNN," in 2018 Fifth International Conference on Emerging Applications of Information Technology (EAIT), pp. 1-4, Kolkata, India, January 2018.

[29] W. Kong and J. Liu, "Technique for image fusion based on nonsubsampled shearlet transform and improved pulsecoupled neural network," Optical Engineering, vol. 52, no. 1, article 017001, 2013.

[30] X. Jin, R. Nie, D. Zhou, Q. Wang, and K. He, "Multifocus color image fusion based on NSST and PCNN," Journal of Sensors, vol. 2016, Article ID 8359602, 12 pages, 2016.

[31] A. Mishra, S. Mahapatra, and S. Banerjee, "Modified FreiChen operator-based infrared and visible sensor image fusion for real-time applications," IEEE Sensors Journal, vol. 17, no. 14, pp. 4639-4646, 2017.

[32] P. Ganasala and A. D. Prasad, "Medical image fusion based on Frei-Chen masks in NSST domain," in 2018 5th International Conference on Signal Processing and Integrated Networks (SPIN), pp. 619-623, Noida, India, February 2018.

[33] S. Singh, R. S. Anand, and D. Gupta, "CT and MR image information fusion scheme using a cascaded framework in ripplet and NSST domain," IET Image Processing, vol. 12, no. 5, pp. 696-707, 2018.

[34] Q. Cao, B. Li, and L. Fan, "Medical image fusion based on GPU accelerated nonsubsampled shearlet transform and $2 \mathrm{D}$ princi- pal component analysis," in 2017 IEEE 2nd International Conference on Signal and Image Processing (ICSIP), pp. 203-207, Singapore, Singapore, August 2017.

[35] V. Bhavana and H. K. Krishnappa, "Multi-modality medical image fusion using discrete wavelet transform," Procedia Computer Science, vol. 70, pp. 625-631, 2015.

[36] M. Haribabu, C. H. Bindu, and K. S. Prasad, "Multimodal medical image fusion of MRI-PET using wavelet transform," in 2012 International Conference on Advances in Mobile Network, Communication and Its Applications, Bangalore, India, August 2012.

[37] A. Wang, H. Sun, and Y. Guan, "The application of wavelet transform to multi-modality medical image fusion," in 2006 IEEE International Conference on Networking, Sensing and Control, pp. 270-274, Ft. Lauderdale, FL, USA, April 2006.

[38] H. M. El-Hoseny, E. S. M. E. Rabaie, W. A. Elrahman, and F. E. A. El-Samie, "Medical image fusion techniques based on combined discrete transform domains," in 201734th National Radio Science Conference (NRSC), Alexandria, Egypt, March 2017.

[39] P. H. Nishan, P. Hill, N. Canagarajah, and D. Bull, "Image fusion using complex wavelets," in Procedings of the British Machine Vision Conference 2002, pp. 487-496, Cardiff, UK., 2002.

[40] R. Singh and A. Khare, "Multimodal medical image fusion using Daubechies complex wavelet transform," in 2013 IEEE Conference on Information and Communication Technologies, Thuckalay, Tamil Nadu, India, April 2013.

[41] N. Kingsbury, "Image processing with complex wavelets," Philosophical Transactions of the Royal Society of London. Series A: Mathematical, Physical and Engineering Sciences, vol. 357, no. 1760, pp. 2543-2560, 1999.

[42] N. Chabi, M. Yazdi, and M. Entezarmahdi, "An efficient image fusion method based on dual tree complex wavelet transform," in 2013 8th Iranian Conference on Machine Vision and Image Processing (MVIP), Zanjan, Iran, September 2013.

[43] S. Sruthy, L. Parameswaran, and A. P. Sasi, "Image fusion technique using DT-CWT," in 2013 International MutliConference on Automation, Computing, Communication, Control and Compressed Sensing (iMac4s), Kottayam, India, March 2013.

[44] K. Padmavathi, M. V. Karki, and M. Bhat, "Medical image fusion of different modalities using dual tree complex wavelet transform with PCA," in 2016 International Conference on Circuits, Controls, Communications and Computing (I4C), pp. 1-5, Bangalore, India, October 2016.

[45] H. Talbi and M. K. Kholladi, "Predator prey optimizer and DTCWT for multimodal medical image fusion," in 2018 International Symposium on Programming and Systems (ISPS), pp. 1-6, Algiers, Algeria, April 2018.

[46] A. Krizhevsky, I. Sutskever, and G. E. Hinton, "Image net classification with deep convolutional neural networks," International Conference on Neural Information Processing Systems, vol. 25, pp. 1097-1105, 2012.

[47] Ö. Çiçek, A. Abdulkadir, S. S. Lienkamp, T. Brox, and O. Ronneberger, "3D U-net: learning dense volumetric segmentation from sparse annotation," in Medical Image Computing and Computer-Assisted Intervention - MICCAI 2016, Springer, 2016.

[48] O. Ronneberger, "Invited talk: U-net convolutional networks for biomedical image segmentation," in Bildverarbeitung für die Medizin 2017, Springer Vieweg, 2017. 
[49] F. Milletari, N. Navab, and S. A. Ahmadi, "V-net: fully convolutional neural networks for volumetric medical image segmentation," in 2016 Fourth International Conference on 3D Vision (3DV), Stanford, CA, USA, October 2016.

[50] G. Balakrishnan, A. Zhao, M. R. Sabuncu, J. Guttag, and A. V. Dalca, "VoxelMorph: a learning framework for deformable medical image registration," IEEE Transactions on Medical Imaging, vol. 38, no. 8, pp. 1788-1800, 2019.

[51] Y. Hu, M. Modat, E. Gibson et al., "Weakly-supervised convolutional neural networks for multimodal image registration," Medical Image Analysis, vol. 49, pp. 1-13, 2018.

[52] X. Yang, R. Kwitt, M. Styner, and M. Niethammer, "Quicksilver: Fast predictive image registration - A deep learning approach," Neuroimage, vol. 158, pp. 378-396, 2017.

[53] Y. Liu, X. Chen, H. Peng, and Z. Wang, "Multi-focus image fusion with a deep convolutional neural network," Information Fusion, vol. 36, pp. 191-207, 2017.

[54] O. Ronneberger, P. Fischer, and T. Brox, "U-net: convolutional networks for biomedical image segmentation," in Medical Image Computing and Computer-Assisted Intervention - MICCAI 2015, Springer, 2015.

[55] G. Zeng, X. Yang, J. Li, L. Yu, P.-A. Heng, and G. Zheng, “3D U-net with multi-level deep supervision: fully automatic segmentation of proximal femur in 3D MR images," in Machine Learning in Medical Imaging, Springer, 2017.

[56] M. Z. Alom, C. Yakopcic, M. Hasan, T. M. Taha, and V. K. Asari, "Recurrent residual U-Net for medical image segmentation," Journal of Medical Imaging, vol. 6, no. 1, 2019.

[57] L. Yu, C. Xun, J. Cheng, and P. Hu, "A medical image fusion method based on convolutional neural networks," in 2017 20th International Conference on Information Fusion (Fusion), Xi'an, China, July 2017.

[58] S. Zagoruyko and N. Komodakis, "Learning to compare image patches via convolutional neural networks," in 2015 IEEE Conference on Computer Vision and Pattern Recognition (CVPR), Boston, MA, USA, June 2015.

[59] X. Liang, P. Hu, L. Zhang, J. Sun, and G. Yin, "MCFNet: multilayer concatenation fusion network for medical images fusion," IEEE Sensors Journal, vol. 19, no. 16, pp. 7107-7119, 2019.

[60] H. Hermessi, O. Mourali, and E. Zagrouba, "Convolutional neural network-based multimodal image fusion via similarity learning in the shearlet domain," Neural Computing and Applications, vol. 30, no. 7, pp. 2029-2045, 2018.

[61] T. D. Vu, H. Journal Yang, V. Q. Nguyen, A. R. Oh, and M. S. Kim, "Multimodal learning using convolution neural network and sparse autoencoder," in 2017 IEEE International Conference on Big Data and Smart Computing (BigComp), Jeju, South Korea, February 2017.

[62] R. Hou, D. Zhou, R. Nie, D. Liu, and X. Ruan, "Brain CT and MRI medical image fusion using convolutional neural networks and a dual-channel spiking cortical model," Medical \& Biological Engineering \& Computing, vol. 57, no. 4, pp. 887900, 2019.

[63] S. Singh and R. S. Anand, "Multimodal neurological image fusion based on adaptive biological inspired neural model in nonsubsampled shearlet domain," International Journal of Imaging Systems and Technology, vol. 29, no. 1, pp. 50-64, 2019.

[64] X. Han, "MR-based synthetic CT generation using a deep convolutional neural network method," Medical Physics, vol. 44, no. 4, pp. 1408-1419, 2017.
[65] F. Fan, Y. Huang, L. Wang et al., "A semantic-based medical image fusion approach,” 2019, http://arxiv.org/abs/1906 .00225 .

[66] X. Xia and B. Kulis, W-Net: a deep model for fully unsupervised image segmentation, 2017.

[67] C. K. Chaitanya, G. S. Reddy, V. Bhavana, and G. S. C. Varma, "PET and MRI medical image fusion using STDCT and STSVD," in 2017 International Conference on Computer Communication and Informatics (ICCCI), Coimbatore, India, January 2017.

[68] A. Saboori and J. Birjandtalab, "PET-MRI image fusion using adaptive filter based on spectral and spatial discrepancy," Signal, Image and Video Processing, vol. 13, no. 1, article 1338, pp. 135-143, 2019.

[69] F. Shabanzade and H. Ghassemian, "Combination of wavelet and contourlet transforms for PET and MRI image fusion," in 2017 Artificial Intelligence and Signal Processing Conference (AISP), Shiraz, Iran, October 2017.

[70] V. Bhavana and H. K. Krishnappa, "Fusion of MRI and PET images using DWT and adaptive histogram equalization," in 2016 International Conference on Communication and Signal Processing (ICCSP), Melmaruvathur, India, April 2016.

[71] H. R. Shahdoosti and A. Mehrabi, "MRI and PET image fusion using structure tensor and dual ripplet-II transform," Multimedia Tools and Applications, vol. 77, no. 17, pp. 2264922670, 2018.

[72] Y. Na, L. Zhao, Y. Yang, and M. Ren, "Guided filter-based images fusion algorithm for CT and MRI medical images," IET Image Processing, vol. 12, no. 1, pp. 138-148, 2018.

[73] C. Prakash, S. Rajkumar, and P. V. S. S. R. Mouli, "Medical image fusion based on redundancy DWT and Mamdani type min-sum mean-of-max techniques with quantitative analysis," in 2012 International Conference on Recent Advances in Computing and Software Systems, pp. 54-59, Chennai, India, April 2012.

[74] J. Yang, Y. Wu, Y. Wang, and Y. Xiong, "A novel fusion technique for CT and MRI medical image based on NSST," in 2016 Chinese Control and Decision Conference (CCDC), pp. 43674372, Yinchuan, China, May 2016.

[75] L. Zhan and X. Ji, "CT and MR images fusion method based on nonsubsampled contourlet transform," in 2016 8th International Conference on Intelligent Human-Machine Systems and Cybernetics (IHMSC), Hangzhou, China, August 2016.

[76] J. M. Patel and M. C. Parikh, "Medical image fusion based on multi-scaling (DRT) and multi-resolution (DWT) technique," in 2016 International Conference on Communication and Signal Processing (ICCSP), pp. 0654-0657, Melmaruvathur, India, April 2016.

[77] H. R. Shahdoosti and Z. Tabatabaei, "MRI and PET/SPECT image fusion at feature level using ant colony based segmentation," Biomedical Signal Processing and Control, vol. 47, pp. 63-74, 2019.

[78] J. Du, W. Li, and B. Xiao, "Fusion of anatomical and functional images using parallel saliency features," Information Sciences, vol. 430-431, pp. 567-576, 2018.

[79] E. Daniel, "Optimum wavelet-based homomorphic medical image fusion using hybrid genetic-grey wolf optimization algorithm," IEEE Sensors Journal, vol. 18, no. 16, pp. 68046811, 2018.

[80] J. Wang, X. Li, Y. Zhang, and X. Zhang, "Adaptive decomposition method for multi-modal medical image fusion," IET Image Processing, vol. 12, no. 8, pp. 1403-1412, 2018. 
[81] S. Li, X. Kang, and J. Hu, "Image fusion with guided filtering," IEEE Transactions on Image Processing, vol. 22, no. 7, pp. 2864-2875, 2013.

[82] J. Du, W. Li, B. Xiao, and Q. Nawaz, "Medical image fusion by combining parallel features on multi-scale local extrema scheme," Knowledge-Based Systems, vol. 113, pp. 4-12, 2016.

[83] Y. Liu, S. Liu, and Z. Wang, "Medical image fusion by combining nonsubsampled contourlet transform and sparse representation," in Chinese Conference on Pattern Recognition, pp. 372-381, Springer, 2014.

[84] S. Das and M. K. Kundu, "NSCT-based multimodal medical image fusion using pulse-coupled neural network and modified spatial frequency," Medical \& Biological Engineering \& Computing, vol. 50, no. 10, pp. 1105-1114, 2012.

[85] Z. Zhu, M. Zheng, G. Qi, D. Wang, and Y. Xiang, “A phase congruency and local Laplacian energy based multi-modality medical image fusion method in NSCT domain," IEEE Access, vol. 7, pp. 20811-20824, 2019.

[86] S. Das and M. K. Kundu, "A neuro-fuzzy approach for medical image fusion," IEEE Transactions on Biomedical Engineering, vol. 60, no. 12, pp. 3347-3353, 2013.

[87] B. K. Shreyamsha Kumar, "Multifocus and multispectral image fusion based on pixel significance using discrete cosine harmonic wavelet transform," Signal, Image and Video Processing, vol. 7, no. 6, pp. 1125-1143, 2013.

[88] B. K. Shreyamsha Kumar, "Image fusion based on pixel significance using cross bilateral filter," Signal, Image and Video Processing, vol. 9, no. 5, pp. 1193-1204, 2015.

[89] J. Du, W. Li, K. Lu, and B. Xiao, "An overview of multi-modal medical image fusion,” Neurocomputing, vol. 215, pp. 3-20, 2016. 